UH

iti Universität Hamburg

DER FORSCHUNG | DER LEHRE | DER BILDUNG

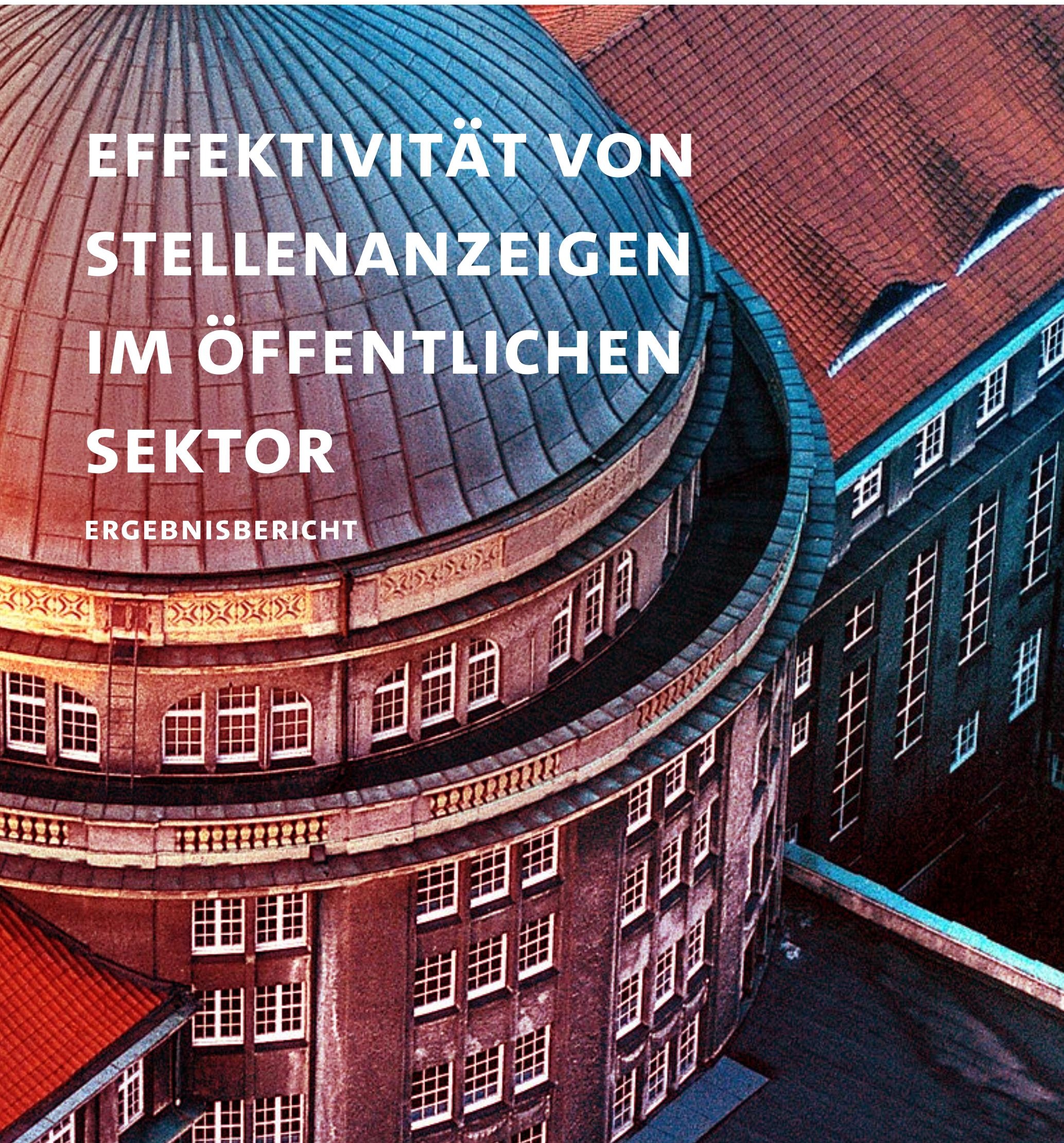




\section{Effektivität von Stellenanzeigen im öffentlichen Sektor}

Prof. Dr. Dominik Vogel (Universität Hamburg)

Prof. Dr. Matthias Döring (Süddänische Universität)

Martin Sievert, M.Sc. (Universität Mannheim)

Stand: 06.12.2021

Autoren:

Prof. Dr. Dominik Vogel, Prof. Dr. Matthias Döring, Martin Sievert, M.Sc.

Mitarbeit:

Jessica Steenbock

Empfohlene Zitierweise: Vogel, D., Döring, M., \& Sievert, M. (2021). Effektivität von Stellenanzeigen im öffentlichen Sektor. Hamburg: Universität Hamburg.

\section{(c) (i)}

Dieses Werk steht unter einer Creative Commons Namensnennung 4.0 Deutschland (CC BY 4.0) Lizenz. Es ist erlaubt, das Werk bzw. den Inhalt zu vervielfältigen, zu verbreiten und öffentlich zugänglich zu machen, Abwandlungen und Bearbeitungen des Werkes bzw. Inhaltes anzufertigen sowie das Werk kommerziell zu nutzen. Bedingung für die Nutzung ist die Angabe der Namen der Autoren. 


\section{Inhaltsverzeichnis}

1 Zusammenfassung 4

2 Ausgangslage und Zielsetzung 5

3 Methodik 7

4 Datengrundlage 8

5 Ausgestaltung von Stellenanzeigen 10

6 Rekrutierungsprozess und -erfolg 12

6.1 Anzahl an Bewerbungen 12

6.2 Besetzung der ausgeschriebenen Stellen 14

6.3 Zufriedenheit mit dem Rekrutierungserfolg 14

6.4 Diversität der Bewerbungen 15

6.5 Rekrutierungsprozess 16

7 Einflussfaktoren auf den Rekrutierungserfolg

7.1 Anzahl an Bewerbungen 20

7.2 Qualität des Pools an Bewerbungen 20

7.3 Qualität der ausgewählten Person 21

8 Handlungsempfehlungen 22

8.1 Wertschätzung 22

8.2 Erreichbarkeit 22

8.3 Professionalität 23

8.4 Sichtbarkeit 23

8.5 Sprache 23

8.6 Inhalte 24

8.7 Prozess 24

9 Fazit 25

10 Forschungsteam 25 


\section{Zusammenfassung}

Die öffentliche Verwaltung in Deutschland ist mit der Herausforderung konfrontiert, in einem stark umkämpften Arbeitsmarkt eine zunehmende Zahl von Fachkräften rekrutieren zu müssen. Die Veröffentlichung von Stellenanzeigen stellt ein zentrales Element bei den Bemühungen des öffentlichen Sektors dar, diesem "War for Talent" zu begegnen. Diese Studie hat sich daher zum Ziel gesetzt, den Rekrutierungserfolg der öffentlichen Verwaltung zu untersuchen und Faktoren zu identifizieren, die Stellenanzeigen und Rekrutierungsbemühungen erfolgreicher machen.

Folgende zentrale Erkenntnisse konnten aus der Studie gewonnen werden:

1. Der Mangel an Fachkräften ist in den technischen und erzieherischen Berufen besonders groß, erfasst aber auch viele andere Bereiche.

2. Verwaltungen, die in den Stellenanzeigen darlegen, wie eine Stelleninhaberin bzw. ein Stelleninhaber in dieser Funktion einen positiven Einfluss auf das Leben anderer ausüben oder zum Gemeinwohl beitragen kann, sind erfolgreicher bei der Rekrutierung. Diese Hinweise in den Stellenanzeigen bezeichnen wir als prosoziale Signale.

3. Diese prosozialen Signale werden bisher kaum genutzt.

4. Auch Signale, die extrinsische Vorzüge einer Stelle, wie Gehalt, Urlaubstage oder sonstige Vergünstigungen betonen, haben einen positiven Effekt auf den Rekrutierungserfolg.

5. Stellenanzeigen werden hauptsächlich auf Stellenportalen für den öffentlichen Dienst veröffentlicht. Die Nutzung von allgemeinen Stellenportalen und Karriere-Portalen (z. B. Xing oder LinkedIn) führt jedoch zu mehr Bewerbungen und vergrößert damit die Auswahl. Eine Verbreitung auf Social-Media erhöht außerdem die Qualität der Bewerbungen.

6. Rekrutierungsprozesse werden von den Verantwortlichen oftmals als unnötig bürokratisch wahrgenommen. Eine starke Bürokratisierung führt außerdem zu einer geringeren Zufriedenheit mit der ausgewählten Person.

Die Erkenntnisse beruhen auf der Analyse von fast 1.900 Stellenanzeigen, die mit einer wissenschaftlichen Befragung der in den Anzeigen genannten Kontaktpersonen verknüpft wurde. Die Datenbasis stellt die Umfangreichste zum Rekrutierungserfolg des öffentlichen Sektors in Deutschland dar.

Ein herzlicher Dank gilt allen, die sich an der Umfrage beteiligt haben, die dieser Studie zugrunde liegt oder unsere Anfrage an diejenigen weitergeleitet haben, die für die Beantwortung der Umfrage am besten geeignet waren. Wir bedanken uns darüber hinaus für zahlreiche Rückmeldungen und das große Interesse an der Studie.

Prof. Dr. Dominik Vogel

Prof. Dr. Matthias Döring

Martin Sievert, M.Sc. 


\section{Ausgangslage und Zielsetzung}

Der öffentliche Dienst sieht sich bei der Rekrutierung von neuem Personal vor zahlreiche Herausforderungen gestellt. Der allgemeine demographische Wandel der Gesellschaft führt zu einer Verringerung des Arbeitskräftepotentials und erhöht dadurch die Konkurrenz des öffentlichen Sektors mit der Privatwirtschaft um qualifiziertes Personal. Dies geschieht vor dem Hintergrund einer ohnehin vorhandenen Knappheit von Fachkräften, die sich insbesondere in den technischen und medizinischen Berufen zeigt, zunehmend aber auch weitere Bereiche erfasst.

Hinzu kommen spezifische Herausforderungen für den öffentlichen Sektor, die bei gleichzeitiger Verknappung von Fachpersonal zu einem erhöhten Bedarf an selbigem führt. Hier ist zum einen der demographische Wandel innerhalb der öffentlichen Verwaltung zu nennen. Durch die Einstellungspolitik der 1990er- und frühen 2000er-Jahre liegt das Durchschnittsalter der Beschäftigten des öffentlichen Dienstes über dem der Erwerbsbevölkerung. Der öffentliche Dienst ist bereits jetzt davon betroffen, dass erhebliche Teile der Beschäftigten in den Ruhestand verabschiedet werden müssen. Dieser Trend wird sich in den kommenden Jahre noch verstärken. Hinzu kommt, dass der Bedarf an neuem Personal auch durch eine Ausweitung der öffentlichen Aufgaben in den letzten Jahren gestiegen ist. Dies schlägt sich auch in der Gesamtzahl der Beschäftigten im öffentlichen Dienst nieder, die von 4,5 Millionen im Jahr 2009 auf 5,0 Millionen im vergangenen Jahr gestiegen ist.

Zusammengefasst stellen diese Trends den öffentlichen Sektor vor die Herausforderung, in einer oftmals schwierigen Arbeitsmarktlage eine zunehmende Anzahl von Beschäftigten rekrutieren zu müssen. Dabei ist es nicht nur im Interesse der Verwaltungen selbst, sondern auch der Gesamtgesellschaft, dass der öffentliche Dienst diesen Personalbedarf mit möglichst kompetentem und motiviertem Personal decken kann.

Diese Studie setzt an diesem Punkt an und untersucht, wie die öffentliche Verwaltung das Ziel, qualifiziertes und motiviertes Personal zu finden, besser erreichen kann. Sie konzentriert sich dabei auf die Stellenanzeigen, die von Verwaltungen veröffentlicht werden, um offene Stellen zu besetzen. Diese Stellenanzeigen spielen eine elementare Rolle bei der Personalrekrutierung im öffentlichen Dienst. Sie sind in der Regel das zentrale Instrument, um potentielle Bewerber:innen auf eine Stelle aufmerksam zu machen und zu einer Bewerbung zu motivieren. Stellenanzeigen sind auch deshalb von großer Bedeutung, da direktere Formen des Personalmarketings, wie die Direktansprache potentieller Kandidat:innen, aufgrund gesetzlicher Regelungen höchstens komplementär zur Verfügung stehen. Finanzielle Einschränkungen und hohe Auslastungen des Personalmanagements stellen zudem weitere Hürden beim Einsatz alternativer Personalmarketinginstrumente dar.

Die Gestaltung und Veröffentlichung von Stellenanzeigen ist somit das zentrale Element von Rekrutierungsbemühungen im öffentlichen Sektor. Diese
Fachkräftemangel trifft auf erhöhten Personalbedarf

Ausgestaltung von Stellenanzeigen als Möglichkeit zur Steigerung des Rekrutierungserfolgs 
Studie verfolgt daher zwei Ziele. Zum einen soll sie einen Überblick über den Einsatz von Stellenanzeigen im öffentlichen Sektor bieten und dabei systematische Daten zu Bewerbungslage und Rekrutierungserfolg liefern. Zum anderen soll untersucht werden, ob sich Charakteristika von Stellenanzeigen sowie von Verwaltungen identifizieren lassen, die mit einem höheren Rekrutierungserfolg einhergehen.

Der vorliegende Ergebnisbericht soll einen Überblick über die im Rahmen der Studie gewonnenen Erkenntnisse bieten. Er führt zunächst in die Methodik der Studie ein, um anschließend die teilnehmenden Verwaltungen und die vorliegenden Daten zu beschreiben. Im Anschluss wird ein Überblick über den Rekrutierungsprozess und den Erfolg der öffentlichen Verwaltung bei der Rekrutierung gegeben, um dann vorzustellen, welche Faktoren den Rekrutierungserfolg beeinflussen. Der Bericht schließt mit Handlungsempfehlungen für den öffentlichen Sektor. 


\section{Methodik}

Das Forschungsprojekt fußt auf zwei Datenquellen, die miteinander kombiniert wurden. Dies sind zum einen Stellenanzeigen, die auf bund.de oder einer verknüpften Seite veröffentlicht wurden und zum anderen Daten einer Befragung, welche von den in der Ausschreibung genannten Kontaktpersonen ausgefüllt wurde. Damit ist es unseres Wissens nach das erste Projekt, das den Inhalt von Stellenanzeigen mit Daten über die Rekrutierungsprozesse der ausschreibenden Verwaltungen kombiniert.

Die Stellenanzeigen wurden automatisiert im Zeitraum zwischen Juni 2020 und März 2021 vom Stellenportal auf bund.de heruntergeladen und lokal in einer Datenbank gespeichert. Die Anzeigen wurden von den Behörden entweder direkt auf bund.de veröffentlicht oder stammen von einem Portal, das mit bund.de verknüpft ist. Dies sind insbesondere Interamt und das Stellenportal des Landes Nordrhein-Westfalen (Karriere.NRW). Auf diesem Wege konnten 58.668 Stellenanzeigen für die weitere Analyse gespeichert werden.

Um über den reinen Inhalt der Stellenanzeigen hinaus Erkenntnisse gewinnen zu können, wurde eine wissenschaftliche Umfrage entwickelt, die die Datenbasis um Informationen und Einschätzungen zum Rekrutierungsprozess und dem Rekrutierungserfolg des einzelnen Stellenbesetzungsverfahrens erweitern sollte. Da die Umfrage in Form einer effizienten Onlinebefragung durchgeführt werden sollte, wurden alle Stellenanzeigen aussortiert, bei denen kein E-Mailkontakt angegeben war. Dies reduzierte den Umfang der Datenbank auf 33.642 Anzeigen. Um die Belastung für die einzelnen Verwaltungen so gering wie möglich zu halten, wurden die Anzeigen nach den angegebenen E-Mailadressen gruppiert und im Falle von Mehrfachnennungen jeweils eine Anzeige zufällig ausgewählt. Auf diesem Wege konnten 7.026 Kontaktpersonen per E-Mail angeschrieben und um eine Teilnahme an der Befragung gebeten werden.

Die Umfrage wurde zwischen April und Juli 2021 durchgeführt. Von den 7.026 Angeschriebenen konnten 215 nicht (mehr) unter der genannten EMailadresse erreicht werden. Von den verbleibenden Kontakten haben 1.888 und damit $27,8 \%$ an der Befragung teilgenommen.

Bei der Durchführung der Studie wurde auf einen umfassenden Datenschutz Wert gelegt. Die Teilnehmenden wurden vor der Befragung über die zu erhebenden Daten sowie ihre Rechte hingewiesen und haben ihr Einverständnis für die Erhebung und Verarbeitung der Daten gegeben. Zur Datenerhebung wurde eine Software genutzt, die von der Universität Hamburg auf eigenen Servern betrieben wird. Nach Abschluss der Befragung wurden die Daten aus dem System gelöscht und auf einem lokalen System der Universität Hamburg gespeichert, auf das nur der Projektleiter Prof. Dr. Dominik Vogel Zugriff hat, der auch die Datenauswertung vornahm. Die Umfragedaten wurden, wie angekündigt, lokal mit den Stellenanzeigen verknüpft. Zur Steigerung des Datenschutzes wurden anschließend die hinterlegten Kontaktdaten gelöscht.
Einzigartige Kombination von Stellenanzeigen und Umfragedaten

Fast 60.000 Stellenanzeigen von bund.de analysiert

Umfrage mit fast 1.900 Teilnehmenden

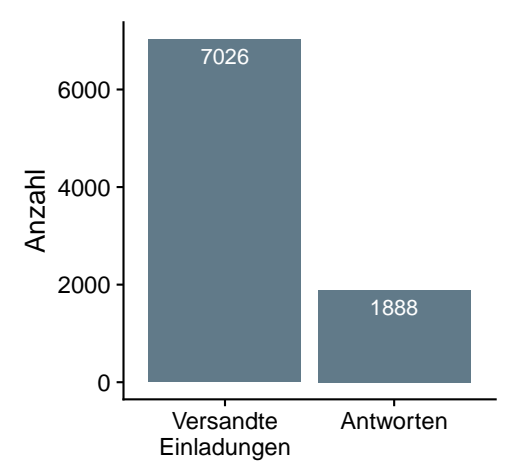

Abbildung 1: Versandte Einladungen zur Umfrage und Rücklauf. 


\section{Datengrundlage}

Die Daten, die dieser Studie zugrunde liegen, spiegeln die große Vielfalt der öffentlichen Verwaltung in Deutschland wider (s. Abbildung 2). Entsprechend der Größe des öffentlichen Dienstes auf den einzelnen staatlichen Ebenen, stammt der größte Teil der Antworten von Landesbehörden (38,5\%), gefolgt von kommunalen Organisationen (35,6\%). Der Bund macht einen Anteil von 12,2\% aus. Die Sozialversicherungsträger haben einen Anteil von 1,3\%. 12,4\% sind anderen Bereichen zuzuordnen.

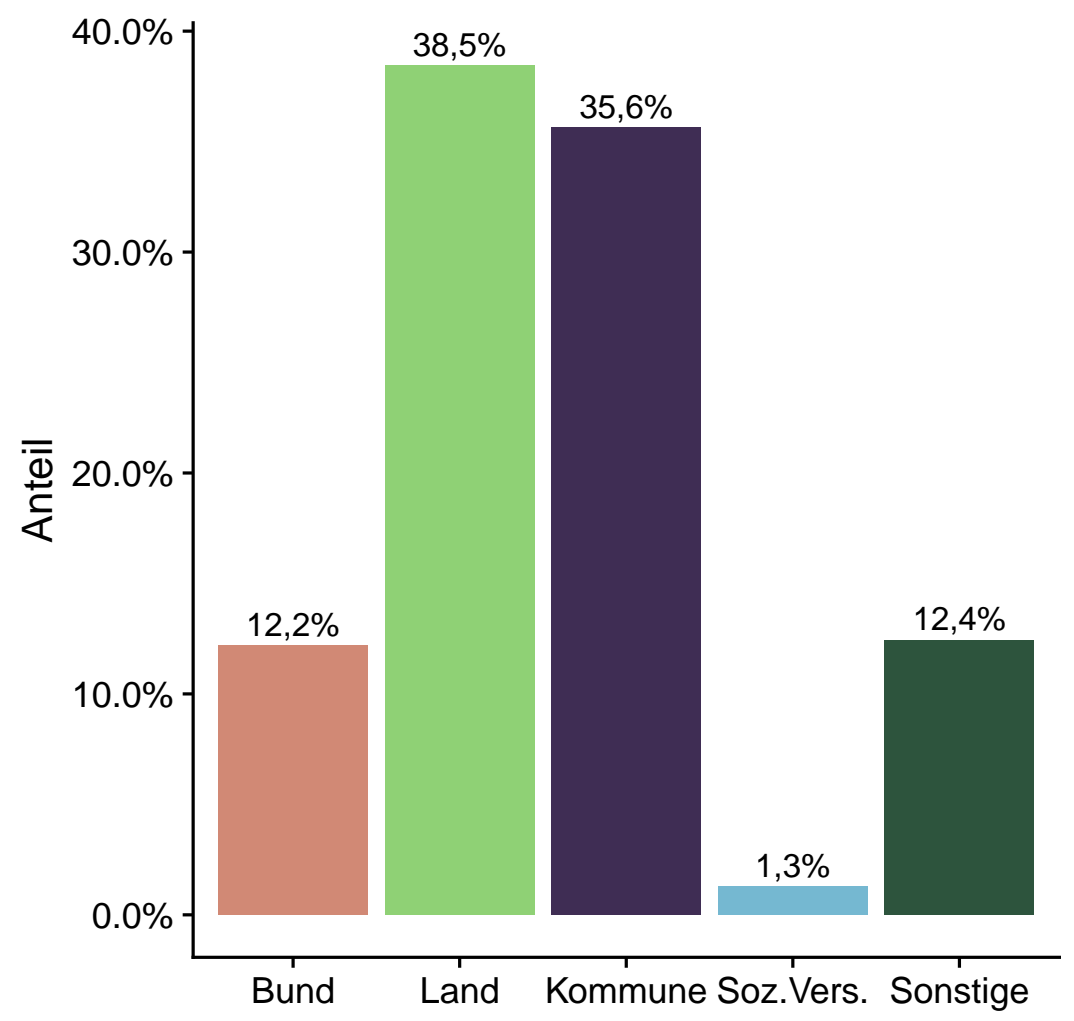

Die in den Stellenanzeigen beworbenen Tätigkeiten sind ebenfalls breit gestreut. Klassische Verwaltungs- und Bürotätigkeiten machen 26,2\% aus, gefolgt von Wissenschaft und Technik (9,3\%), Erziehung und Bildung (8,8\%) und technischer Verwaltung $(6,4 \%)$. Wie in Abbildung 3 zu sehen, liegen die übrigen Bereiche bei jeweils unter vier Prozent. Hinzu kommt ein Anteil von 32,2\%, der sich nicht in die von bund.de vorgegebenen Kategorien einordnen lässt.

Abbildung 2: Teilnehmende nach staatlicher Ebene. klassische Verwaltungs- und Bürotätigkeiten gesucht

Am häufigsten werden Beschäftigte für 


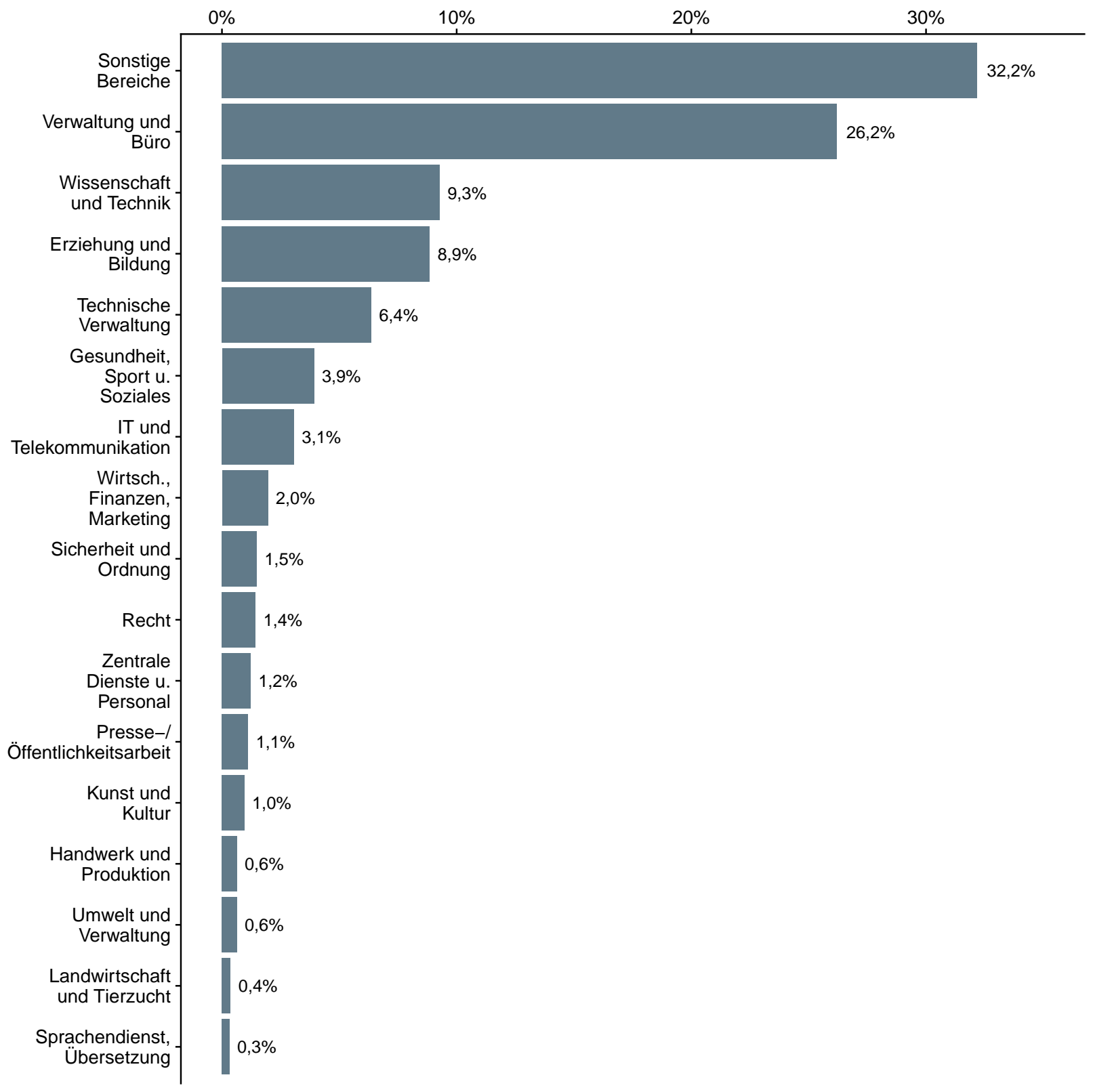

Abbildung 3: Tätigkeitsfelder, in denen Beschäftigte gesucht wurden. 


\section{Ausgestaltung von Stellenanzeigen}

In einem ersten Schritt haben wir den Inhalt der Stellenanzeigen untersucht. Dabei haben wir uns insbesondere darauf konzentriert, welche Signale die Anzeige bezüglich verschiedener Motivationen von Bewerber:innen beinhalten. Mit Motivationssignalen meinen wir Aussagen in den Anzeigen, die entweder die extrinsische, intrinsische oder prosoziale Motivation von potentiellen Bewerber:innen anspricht.

Extrinsische Motivation speist sich aus externen Konsequenzen der Erledigung der Arbeit. Diese können positiv sein, wie zum Beispiel das Gehalt, ein Lob oder Anerkennung für eine Leistung. Extrinsische Motivation kann aber auch aus der Vermeidung negativer Konsequenzen, wie Tadel oder eine Entlassung, entstehen. In beiden Fällen wird die Arbeit als Mittel zur Erreichung eines Zwecks gesehen.

Intrinsische Motivation entsteht hingegen aus der Arbeit selbst. Menschen sind dadurch motiviert, dass innen die Aufgabe selbst Freude bereitet oder sie herausfordert. Zentrale Aspekte können dabei Autonomie bei der Aufgabenerfüllung, Vielfältigkeit der Aufgabe oder schlicht Begeisterung für eine Aufgabe sein.

Prosoziale Motivation entspringt einem Gefühl, mit einer Tätigkeit einen positiven Einfluss auf das Leben anderer haben zu können. Dies können Kolleg:innen, Kund:innen oder Bürger:innen sein, denen man mit der eigenen Tätigkeit helfen kann. Auch die Möglichkeit, einen positiven Beitrag zum Gemeinwohl leisten zu können, kann prosoziale Motivation auslösen.

Um zu erfassen, wie häufig die jeweiligen Anreize genannt wurden, haben wir eine Liste mit Suchbegriffen für jede der drei Arten von Motivationssignalen generiert. Hierzu haben wir stichprobenartig 150 Stellenanzeigen gesichtet und alle Schlüsselbegriffe erfasst. Diese Liste wurde durch Begriffe ergänzt, die aus wissenschaftlichen Definitionen und Fragebögen für die jeweiligen Motive stammen. Die finale Liste umfasste insgesamt 408 Suchbegriffe.

Abbildung 4 visualisiert die Ergebnisse dieser Bemühungen. Sie zeigt wie häufig die extrinsischen, intrinsischen und prosozialen Signale in den Stellenanzeigen identifiziert werden konnten. Es zeigt sich ein starkes Übergewicht der extrinsischen Signale. In einer Stellenanzeige wurden im Schnitt 8,3 extrinsische Signale genannt. Lediglich in $8,1 \%$ der Stellenanzeigen fanden sich keine solchen Signale. Der Maximalwert lag hingegen bei 43 extrinsischen Signalen in einer Anzeige.

Intrinsische Signale sind weniger häufig zu finden. Im Durchschnitt enthält eine Stellenanzeige 1,6 intrinsische Signale. Bei 26,4\% der Anzeigen ist kein intrinsisches Signal zu finden.

Noch seltener sind prosoziale Signale. Im Durchschnitt enthält eine Stellenanzeige lediglich 0,3 prosoziale Signale. Ein Großteil der Anzeigen (78,1\%) enthält kein prosoziales Signal und auch in der Maximalausprägung sind bei einer Stellenanzeige sechs prosoziale Signale zu beobachten. Ein deutlicher
Welche Motivationsformen werden in den Anzeigen angesprochen?

Extrinsische Motivation

Intrinsische Motivation

Prosoziale Motivation

Wörterbuch mit über 400 Stichworten

Extrinsische Motive werden besonders häufig genannt

Prosoziale Motive werden kaum erwähnt 
Unterschied zu den extrinsischen und auch den intrinsischen Signalen.

Es ist selbstverständlich einfacher, eine Vielzahl extrinsischer Anreize in einer Stellenanzeige darzustellen. Die Vielfältigkeit möglicher Motive und die Vertrautheit mit derartigen Signalen machen es leichter, extrinsische Aspekte zu betonen (bspw. Anzahl der Urlaubstage, Besoldung bzw. Entgelt, zusätzliche Vergünstigungen). Dass der Großteil der untersuchten Stellenanzeigen keinerlei prosoziale Anreize hervorstellt, überrascht dennoch. Ist doch der prosoziale Aspekt einer Beschäftigung im öffentlichen Sektor ein starkes Abgrenzungsmerkmal gegenüber anderen Arbeitgebern und wird immer wieder betont, wenn es um die Vorzüge des öffentlichen Dienstes geht.
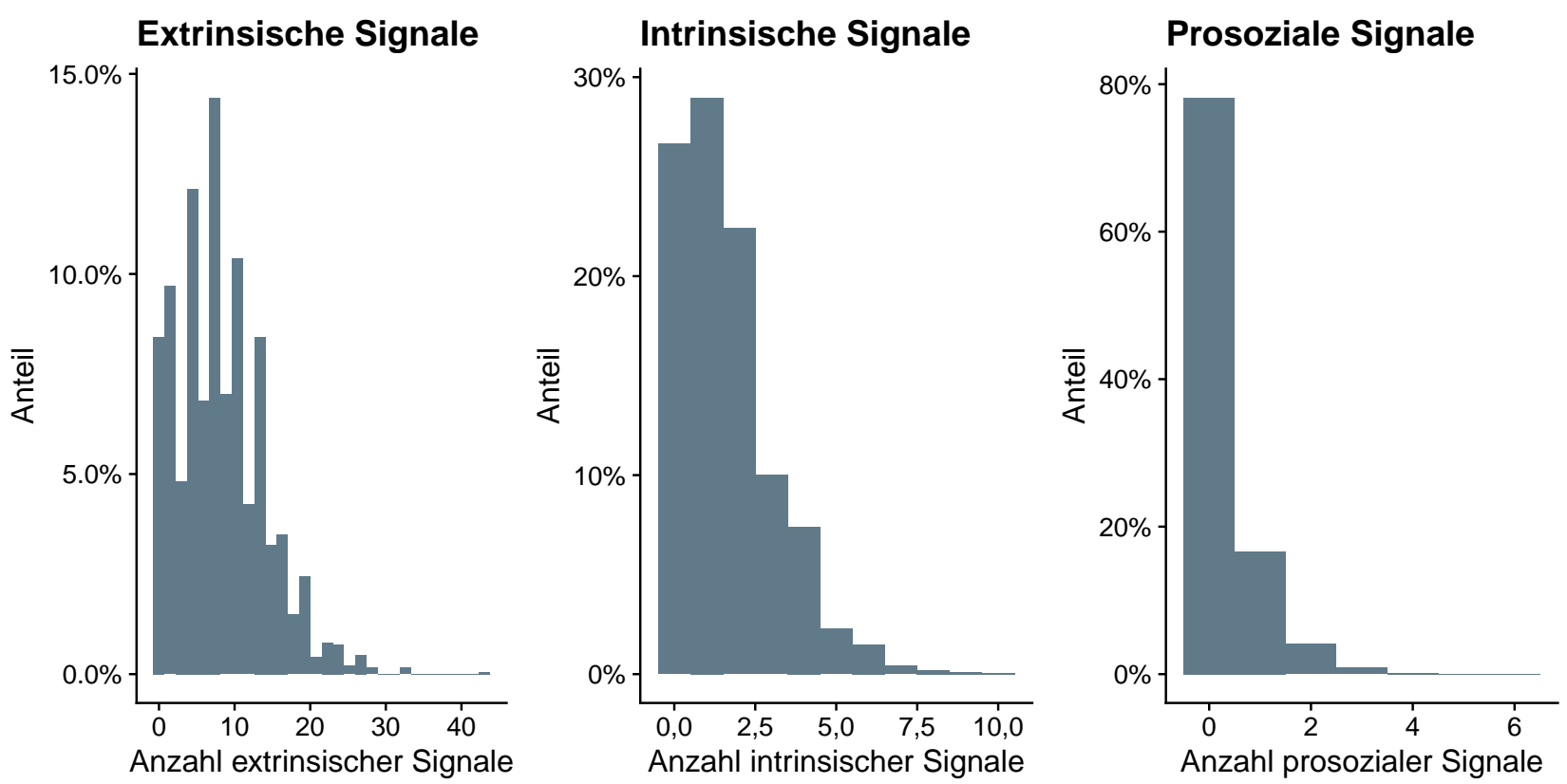

Abbildung 4: Verteilung von extrinsischen, intrinsischen und prosozialen Motivationssignalen in den Stellenanzeigen. 


\section{Rekrutierungsprozess und -erfolg}

In unserer Studie war es uns besonders wichtig, zu erfassen, wie erfolgreich die verschiedenen Verwaltungen bei ihren Einstellungsprozessen sind. Erfolg ist jedoch sehr schwer zu erfassen. Wir haben uns daher auf drei Faktoren konzentriert, die in der Wissenschaft als Ausprägungen von Rekrutierungserfolg anerkannt und über verschiedene Verwaltungen hinweg vergleichbar sind. Dies sind:

- Die Anzahl an erhaltenen Bewerbungen

- Die Qualität der Bewerbungen

- Die Qualität der ausgewählten Person

Die Bedeutung der Anzahl der Bewerbungen ergibt sich aus dem Fakt, dass es - bei ansonsten gleichbleibenden Rahmenbedingungen - wahrscheinlicher ist, eine geeignete Person für eine Stelle zu finden, wenn sich mehr Personen beworben haben. Je größer der Pool an Kandidat:innen ist, desto besser kann eine Verwaltung auswählen. Diese Grundidee wurde auch durch empirische Forschung bestätigt, die zeigt, dass Organisationen, die mehr Bewerbungen anziehen können, langfristig erfolgreicher sind.

Die Qualität der Bewerbungen und die Qualität der ausgewählten Person haben wir als subjektive Einschätzungen der befragten Kontaktpersonen erfasst. Dabei haben wir danach gefragt, wie zufrieden die Kontaktpersonen mit der Qualität der Bewerbungen sowie mit der letztlich ausgewählten Person waren.

\subsection{Anzahl an Bewerbungen}

Wie zu erwarten, erhalten die beteiligten Verwaltungen eine sehr unterschiedliche Anzahl an Bewerbungen. Abbildung 5 gibt einen Überblick darüber. Auf ein Drittel der Stellenanzeigen bewarben sich 20 oder weniger Kandidat:innen. In $44(2,3 \%)$ Fällen gab es lediglich eine Bewerbung. In 6 Fällen haben die Verwaltungen 200 oder mehr Bewerbungen erhalten. In einem Fall betrug die Anzahl der Bewerbungen sogar 600.

Wie im linken Teil von Abbildung 6 zu sehen, stammt der Großteil der Bewerbungen von externen Kandidat:innen. Lediglich 2,7\% aller Bewerbungen entfallen auf interne Bewerbungen. Dem gegenüber steht jedoch ein Anteil von $10,6 \%$ Stellen, die mit internen Kandidat:innen besetzt wurden. Die interne Besetzung von Stellen spielt folglich eine substanzielle Rolle bei der Besetzung der hier untersuchten Stellenanzeigen.
Anzahl an erhaltenen Bewerbungen, Qualität der Bewerbungen und Qualität der ausgewählten Person sind zentrale Elemente von Rekrutierungserfolg

Organisationen, die mehr Bewerbungen erhalten, sind langfristig erfolgreicher

Auf ein Drittel der Anzeigen bewerben sich 20 oder weniger Menschen

Nur drei Prozent der Bewerbungen kommen von internen Kandidat:innen 


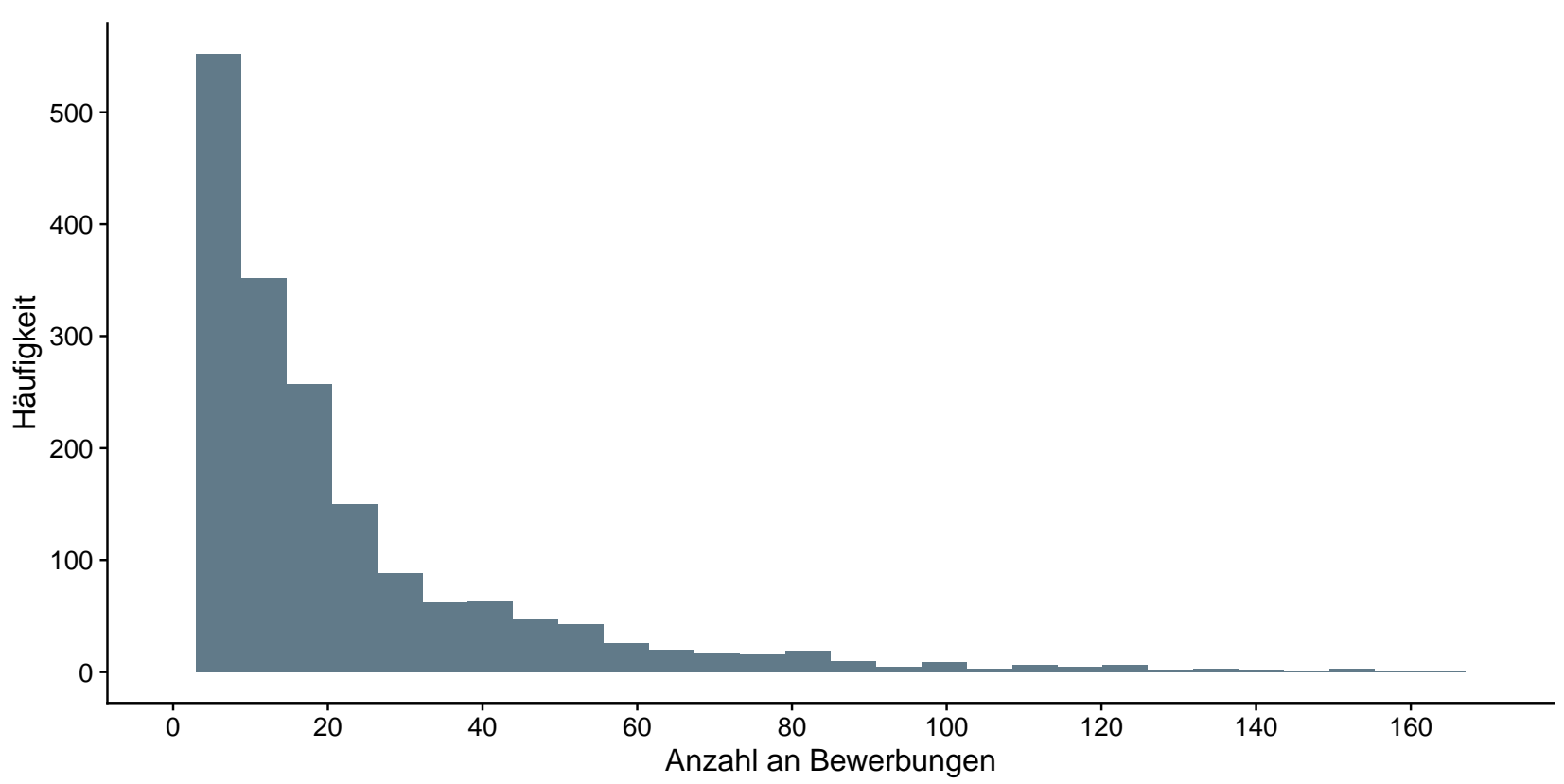

Größer ist die Bandbreite beim Anteil von Bewerbungen von Frauen (rechter Abbildung 5: Verteilung der Anzahl der Bewerbungen. Sechs Fälle mit 200 oder mehr Bewerbungen wurden ausgeblendet.

Teil in Abbildung 6). Dieser beträgt im Mittel 51,3\% und spiegelt damit ungefähr das aktuelle Geschlechterverhältnis im öffentlichen Dienst wider (Frauenanteil $=57 \%$ ). Interessant ist zudem, dass in $11,8 \%$ der Fälle keine Bewerbungen von Frauen eingegangen sind. In diesen Bereichen könnten besondere Bemühungen um Bewerbungen von Frauen möglicherweise zu einer Verbesserung der Bewerbungssituation beitragen. Das andere Extrem besteht aus 128 Stellenanzeigen $(6,8 \%)$, auf die sich ausschließlich Frauen beworben haben.
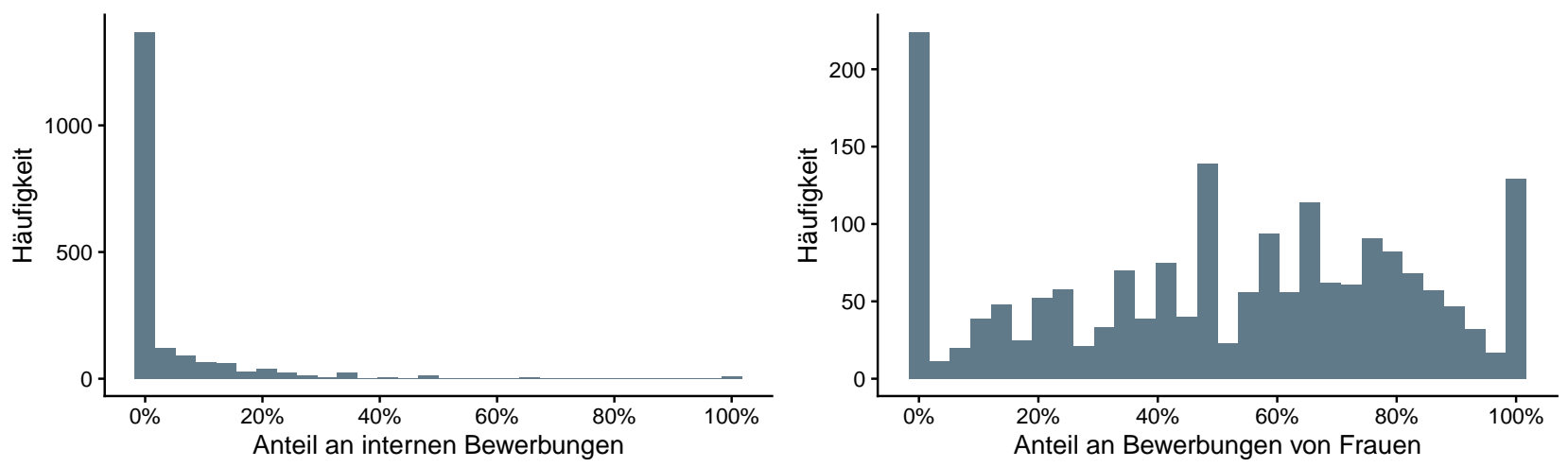

Abbildung 6: Verteilung des Anteils an Bewerbungen von internen Bewerber:innen (links) sowie von Frauen (rechts) an allen Bewerbungen. 


\subsection{Besetzung der ausgeschriebenen Stellen}

Wir haben die Teilnehmenden unserer Studie gefragt, ob die in der jeweiligen Stellenanzeige genannte Position besetzt werden konnte (s. Abbildung 7). Dies war bei 75,3\% der Stellen der Fall. Für eine Nichtbesetzung gab es eine Vielzahl von Gründen. Die Häufigste war, dass das Verfahren derzeit noch läuft. Dies war bei 10,0\% der Ausschreibungen der Fall. Zieht man diese von der Gesamtzahl ab, so beträgt der Anteil der erfolgreichen Besetzungen 83,7\%.

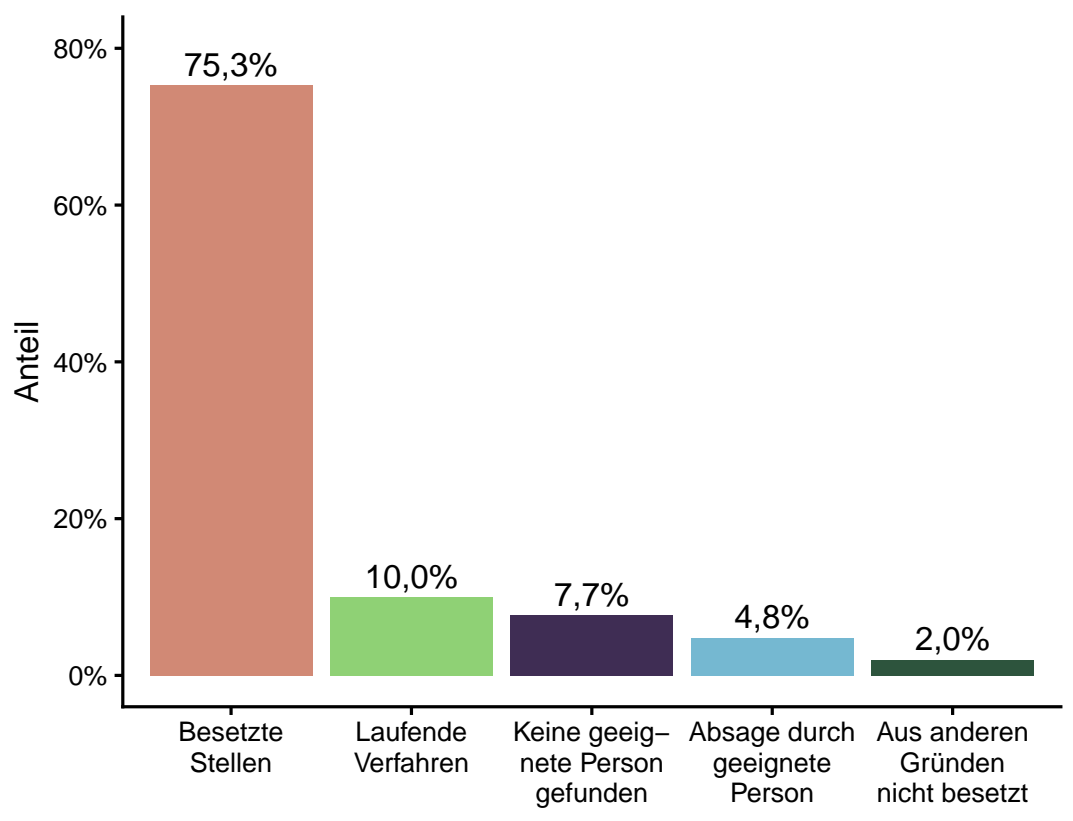

Abbildung 7: Anteil besetzter Stellen an allen Stellen sowie Anteile verschiedener Gründe für die Nichtbesetzung von Stellen.

84 Prozent der Stellen konnten besetzt werden
Auffällig ist hierbei auch, dass einige der Verfahren zum Zeitpunkt der Erhebung noch andauerten, obwohl die Bewerbungsfrist bereits seit einiger Zeit abgelaufen war. In 55,0\% der Fälle lag der Bewerbungsschluss zwar weniger als drei Monate zurück, 21,2\% der laufenden Verfahren hatten das Bewerbungsende jedoch bereits um mehr als sechs Monate überschritten. Der Maximalwert lag bei 320 Tagen.

\subsection{Zufriedenheit mit dem Rekrutierungserfolg}

Abgesehen von der Quantität an Bewerbungen berücksichtigen wir außerdem die Qualität dieser auf Basis der subjektiven Einschätzung der rekutierenden Verwaltung. Dies gilt insbesondere für die Qualität des Pools an Bewerber:innen und der letztlich ausgewählten Person. Wir haben die Teilnehmenden dieser Studie daher gefragt, wie zufrieden sie mit einzelnen Elementen ihres Rekrutierungserfolgs sind. Dabei haben wir folgende Faktoren berücksichtigt:
Besetzungsverfahren dauern mitunter äußegewöhnlich lange

Nicht nur Anzahl, sondern vor allem die Qualität der Bewerbungen ist von großer Bedeutung 
- Zufriedenheit mit der Anzahl an Bewerbungen

- Zufriedenheit mit der Qualität der Bewerbungen

- Zufriedenheit mit der ausgewählten Person (wenn Verfahren bereits abgeschlossen waren)

Die Ergebnisse finden sich in Abbildung 8. Es zeigt sich, dass die Mehrheit der Befragten eher zufrieden mit diesen drei Aspekten ist. Der Anteil der

Die Mehrheit ist eher oder sehr zufrieden mit ihrem Rekrutierungserfolg Zufriedenen beträgt bei der Anzahl der Bewerbungen 49\% gegenüber 33\% Unzufriedenen. Ähnlich sieht die Verteilung bei der Qualität des Pools an Bewerbungen aus. Hier beträgt das Verhältnis 51\% Zufriedene zu $30 \%$ Unzufriedenen. Noch positiver fällt das Ergebnis bei der Zufriedenheit mit der ausgewählten Person aus. Hier sind lediglich $7 \%$ unzufrieden, während $90 \%$ zufrieden sind. An dieser Stelle muss jedoch berücksichtigt werden, dass lediglich diejenigen eine Einschätzung der ausgewählten Person abgeben konnten, die auch in der Lage waren, die Stelle zu besetzen. Wie in Abschnitt 7 aufgezeigt, ist dies zum Zeitpunkt der Befragung nur bei 75,3\% der Fall gewesen. Man kann also sagen, wenn eine Stelle besetzt werden konnte, sind die Verwaltungen überwiegend zufrieden mit der ausgewählten Person. Die Auswertung zeigt jedoch, dass 7,7\% den Besetzungsprozess abgebrochen haben, weil sie keine geeignete Person für die Stelle gefunden und damit spätere Unzufriedenheit vermieden haben. Hinzu kommen 4,8\% der Verfahren, bei denen zwar eine geeignete Person gefunden wurde, diese die Stelle jedoch nicht angetreten hat (siehe Abbildung 7).

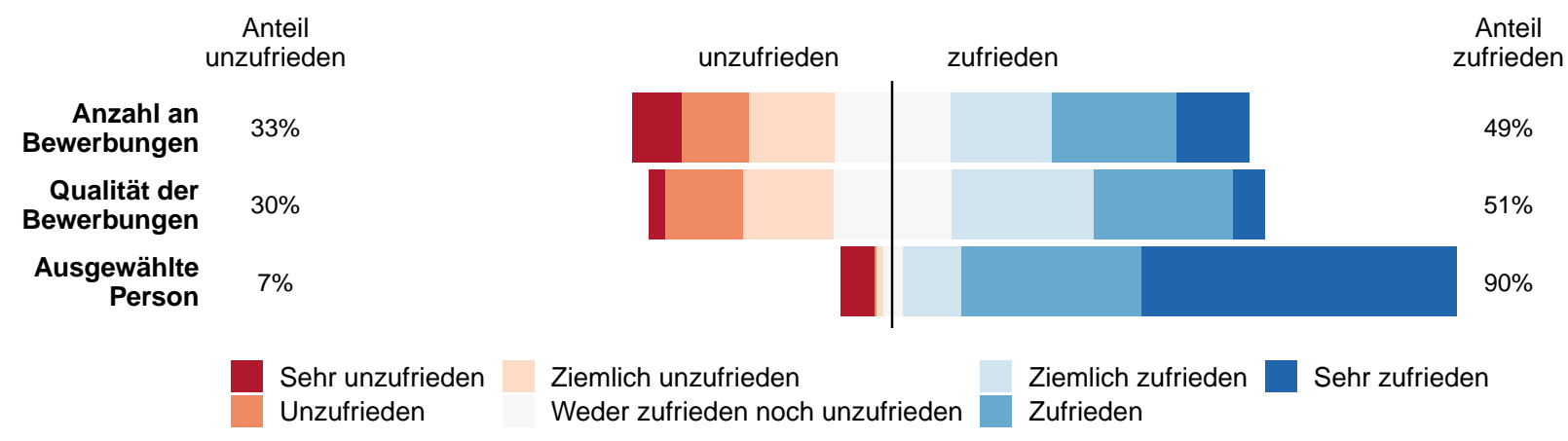

Abbildung 8: Zufriedenheit mit verschiedenen Aspekten des Rekrutierungserfolgs.

\subsection{Diversität der Bewerbungen}

In einem kompetitiven Arbeitsmarkt ist es für Verwaltungen wichtig, die volle Bewerbungen im Durchschnitt wenig divers Bandbreite potentieller Bewerber:innen anzusprechen. Die Forschung zeigt darüber hinaus, dass vielfältig zusammengesetzte Teams erfolgreicher sind als homogene Teams. Wir haben die Teilnehmenden daher gefragt, für wie divers sie den Pool an Bewerbungen empfunden haben. Die Ergebnisse sind in 
Abbildung 9 dargestellt. Lediglich mit Hinblick auf das Alter ist die Mehrheit der Befragten der Meinung, dass ein hohes Maß an Diversität zu beobachten war. Mit Blick auf das Geschlecht und die ethnische Herkunft ist die Mehrheit der Befragten hingegen der Meinung, dass das Feld der Bewerber:innen wenig divers war.

Diese Ergebnisse decken sich mit den bereits vorgestellten Zahlen zur Anzahl an Bewerbungen von Frauen. Hier hatten insgesamt fast ein Fünftel der Befragten angegeben, ausschließlich Bewerbungen von Frauen oder Männern erhalten zu haben.

Vor dem Hintergrund der als relativ gering empfundenen Diversität an Kandidat:innen empfiehlt es sich für den öffentlichen Dienst, bereits angestoßene Bemühungen zur Gewinnung von Frauen in von Männern geprägten Berufen bzw. von Männern in von Frauen geprägten Berufen sowie zur Ansprache von Menschen mit Migrationsbiographie zu verstärken.

\section{Die Bewerbungen waren sehr divers in Hinblick auf ...}

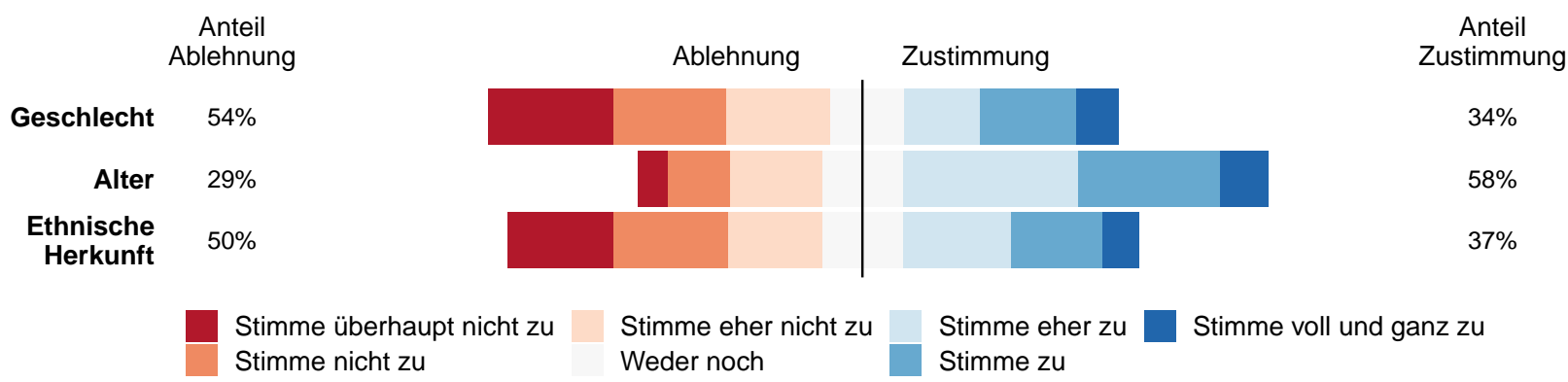

\subsection{Rekrutierungsprozess}

Neben den Ergebnissen zu den Rekrutierungsbemühungen der beteiligten Verwaltungen haben wir uns auch für den Rekrutierungsprozess an sich interessiert. Wir haben die Teilnehmenden deshalb gefragt, wie sie ihre Stellenanzeigen bekannt machen. Dabei haben wir neun verschiedene Möglichkeiten abgefragt. Die Ergebnisse sind in Abbildung 10 zusammengefasst.

Neben der Veröffentlichung in öffentlichen Stellenportalen wie bund.de, Interamt oder Karriere.NRW, die alle Teilnehmenden nutzten, ist die Veröffentlichung auf der Behördenwebsite am gängigsten. Private Stellenportale wie Stepstone oder Indeed und Zeitungsannoncen nutzten ein Drittel der Befragten. Ungefähr ein Viertel der Teilnehmenden verbreitete die Anzeige auch über Social Media und über informelle Kontakte. Noch einmal deutlich seltener werden Karriere-Portale wie Xing oder LinkedIn, Amtsblätter und Newsletter genutzt.

Neben den Marketinginstrumenten haben wir uns auch dafür interessiert, für wie bürokratisch die Beteiligten ihren Rekrutierungsprozess empfinden. Wir

Abbildung 9: Diversität der Bewerber:innen mit Blick auf Alter, Geschlecht und ethnische Herkunft.
Stellenanzeigen werden hauptsächlich auf Stellenportalen für den öffentlichen Sektor und auf der Homepage veröffentlicht

Rekrutierungsprozess wird von Verantwortlichen als relativ bürokratisch wahrgenommen 


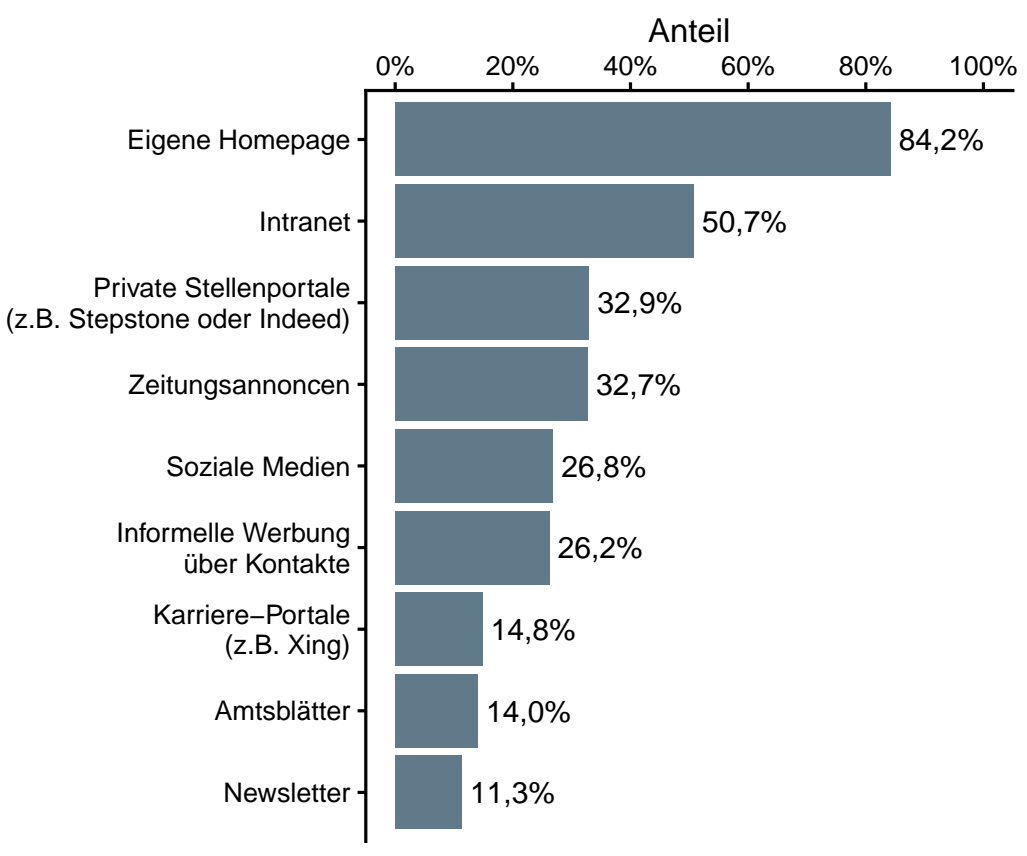

Abbildung 10: Nutzung verschiedener Marketingkanäle.

haben die Teilnehmenden daher gefragt, für wie stark sie den Rekrutierungsprozess durch übermäßige Bürokratie beeinträchtigt sehen. Abbildung 11 stellt die Ergebnisse dar.

Es zeigt sich, dass die Bürokratisierung des Prozesses im Mittel mit 4,5 auf einer Skala von eins bis sieben und damit relativ stark eingeschätzt wird. Lediglich 23\% sind der Meinung, dass der Rekrutierungsprozess eher nicht oder gar nicht durch übermäßige Bürokratie beeinträchtigt wird. Wohingegen $54 \%$ dem eher oder stark zustimmen. Selbstverständlich können Rekrutierungsprozesse in der öffentlichen Verwaltung nicht ohne Regeln und Verfahrensvorschriften auskommen. Die Bandbreite an unterschiedlichen Wahrnehmungen legt jedoch nahe, dass bei manchen Verwaltungen mehr bürokratischer Aufwand verursacht wird, als eigentlich notwendig wäre. Hier können möglicherweise noch Optimierungspotentiale genutzt werden.

\section{Wie stark ist der Rekrutierungsprozess durch übermäßig Bürokratie geprägt?}

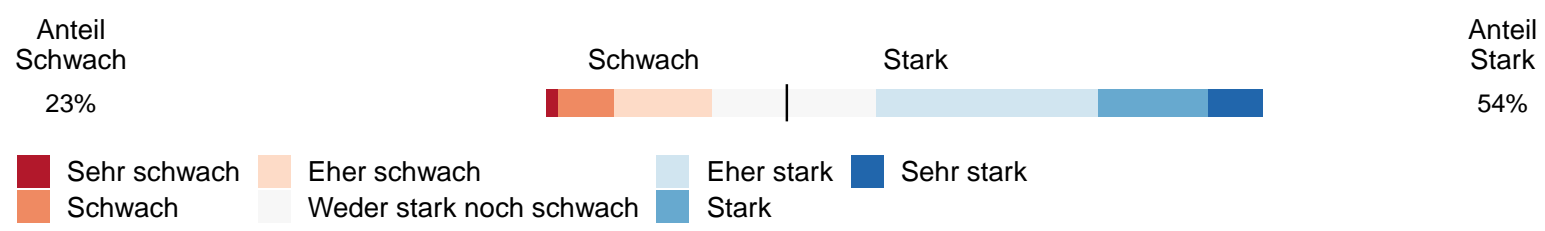




\section{Einflussfaktoren auf den Rekrutierungserfolg}

In diesem Abschnitt möchten wir Ihnen die Ergebnisse unserer statistischen Analysen präsentieren. Wir haben untersucht, welche Faktoren manche Verwaltungen bei der Rekrutierung erfolgreicher machen als andere. Dabei haben wir wieder die bereits erwähnten drei Elemente von Rekrutierungserfolg erfasst:

- Die Anzahl an Bewerbungen

- Die Qualität der Bewerbungen

- Die Qualität der ausgewählten Person

Abbildung 12 präsentiert die Ergebnisse der Regressionsanalysen. Jede der drei Einzelgrafiken stellt dabei das Ergebnis einer Analyse dar. Die erste Grafik stellt die Einflussfaktoren auf die Anzahl der erhaltenen Bewerbungen dar. Die zweite Grafik befasst sich mit Einflussfaktoren auf die Zufriedenheit mit der Qualität des Pools an Bewerbungen, während die letzte Grafik sich den Einflussfaktoren auf die Zufriedenheit mit der ausgewählten Person widmet. Die einzelnen Punkte geben den geschätzten Einfluss eines Faktors auf den jeweiligen Aspekt des Rekrutierungserfolgs an. Dies ist noch einmal in der Grafik rechts auf dieser Seite erläutert. Hier ist der Einfluss einer Ausschreibung aus dem Bericht "IT und Telekommunikation" auf die Anzahl der Bewerbungen dargestellt. Dieser beträgt -21,2 - der Punkt liegt auf der $x$-Achse bei -21,2. Auf Stellenausschriebungen aus dem IT-Bereich gehen also unter Berücksichtigung aller anderen Faktoren durchschnittlich 21,2 Bewerbungen weniger ein als auf Stellen aus dem allgemeinen Verwaltungsdienst.

Die einzelnen Punkte werden von Linien links und rechts des Punktes ergänzt. Diese verdeutlichen die Unsicherheit der Schätzung (das 95\%-Konfidenzintervall). Dies wird ebenfalls in der Grafik rechts deutlich. Der Unsicherheitsbereich hilft auch dabei, die statistische Signifikanz eines Einflussfaktors einzuschätzen. Faktoren, bei denen der Unsicherheitsbereich die eingezeichnete Nulllinie nicht berührt, sind statistisch signifikant. ${ }^{1}$ Dies bedeutet, es ist sehr unwahrscheinlich einen solchen Effekt zu beobachten, wenn in Wirklichkeit gar kein Effekt besteht. Wir können also recht sicher sein, dass wir nicht rein zufällig einen solchen Effekt beobachten. Zur besseren Unterscheidung wurden statistisch signifikante Effekte rot eingefärbt. Schließlich finden sich noch einige Effekte, die in einem blassen Rotton eingefärbt wurden. Bei diesen schließt der Unsicherheitsbereich die Nulllinie zwar ein, jedoch nur sehr knapp. ${ }^{2}$ Wir sind daher nicht sicher, ob ein Einfluss tatsächlich besteht, können dies aber auch nicht gänzlich ausschließen. Bei blau eingefärbten Effekten können wir davon ausgehen, dass sie keinen Einfluss auf den jeweiligen Aspekt des Rekrutierungserfolgs haben.

In den folgenden Abschnitten werden die drei Aspekte des Rekrutierungserfolgs einzeln diskutiert.

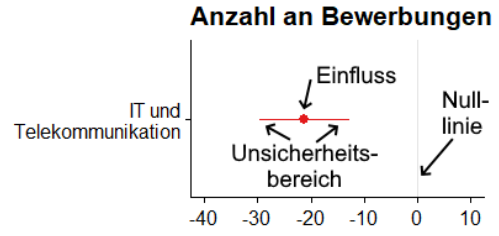

Erläuterung der Ergebnisdarstellung

${ }^{1}$ Der $p$-Wert liegt unter 0.05 .

${ }^{2}$ Diese Effekte weisen einen $p$-Wert zwischen 0.05 und 0.10 auf. 


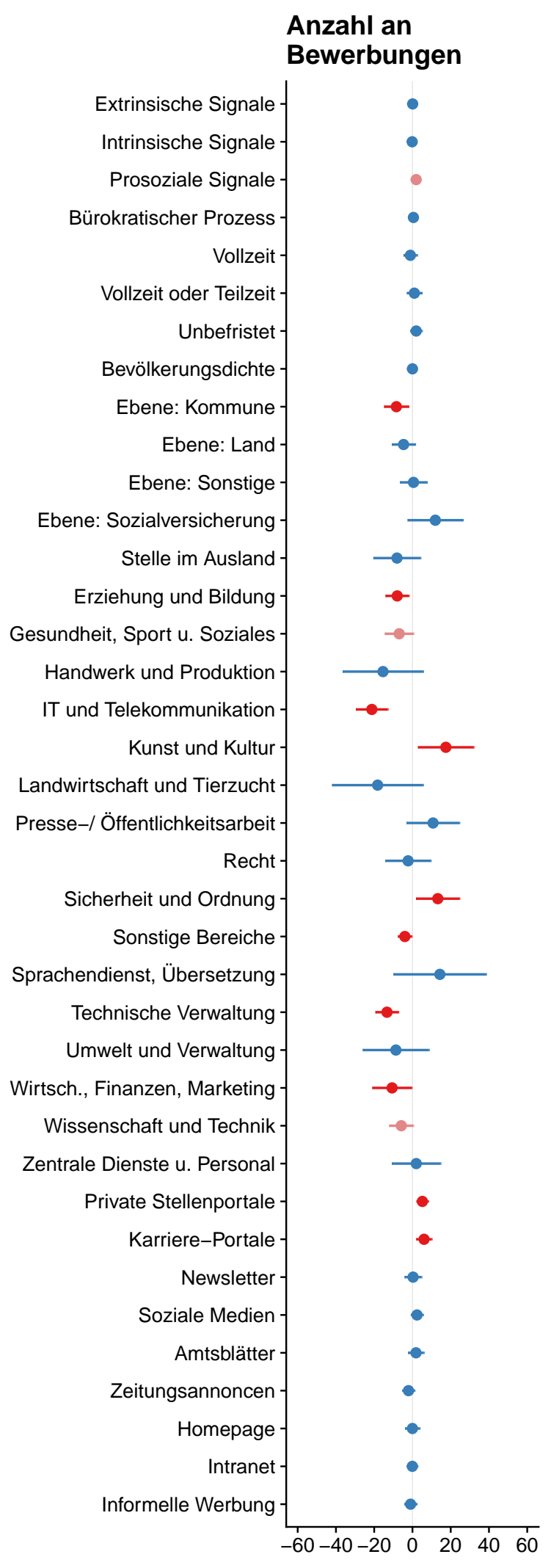

\section{Zufriedenheit mit Qualität Bewerbungen}

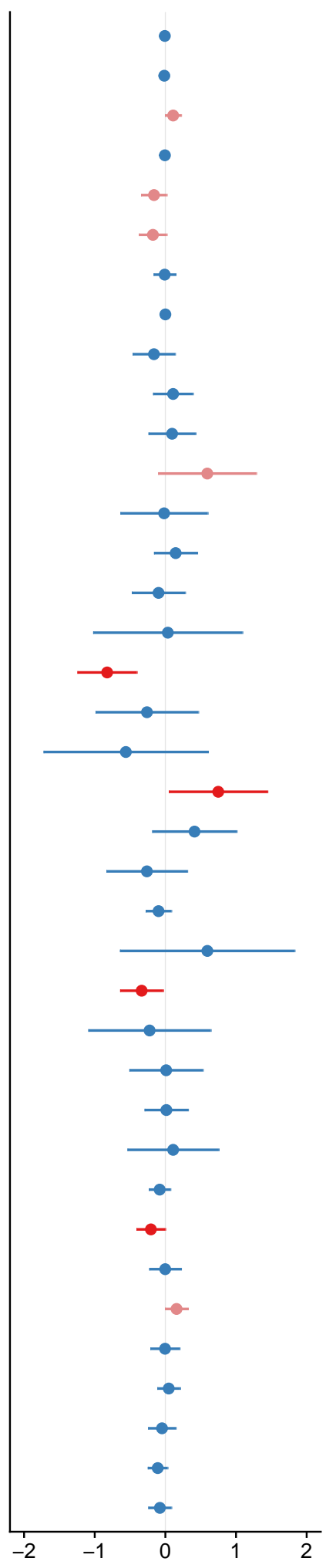

\section{Zufriedenheit mit ausgewählter Person}
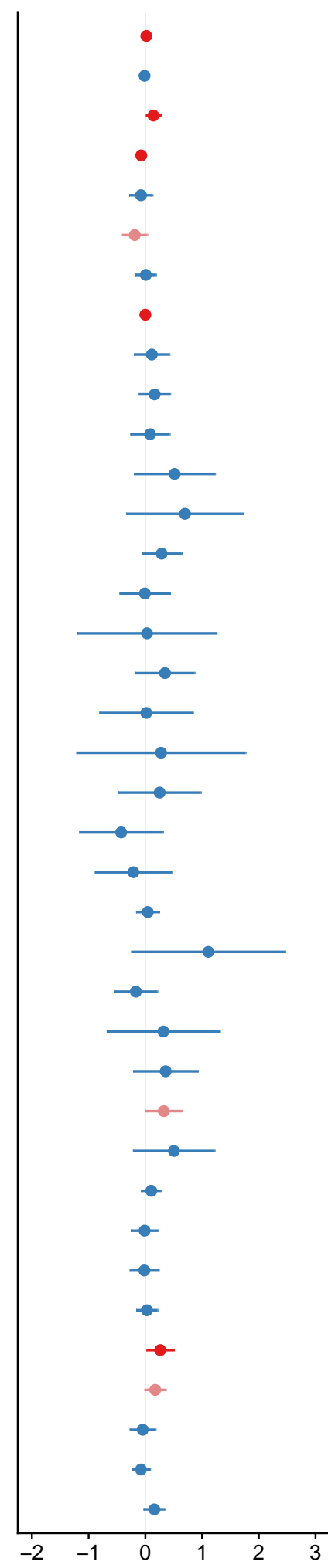

Abbildung 12: Einflussfaktoren auf den Rekrutierungserfolg. Ergebnisse einer hierarchischen Regressionsanalyse. Statistisch signifikante Faktoren sind in rot gefärbt. 


\subsection{Anzahl an Bewerbungen}

Es gibt insgesamt neun Faktoren, bei denen ein statistisch signifikanter Einfluss auf die Anzahl an Bewerbungen festgestellt werden konnte. Bei den Verwaltungsebenen kann beobachtet werden, dass Kommunen signifikant weniger Bewerbungen erhalten als Verwaltungen auf Bundesebene. Dies deckt sich mit Beschreibungen, dass Kommunen besonders stark unter einem Mangel an Fachpersonal leiden.

Darüberhinaus hat auch der Tätigkeitsbereich, in dem eine Verwaltung neues Personal sucht einen Einfluss. Für die statistische Analyse dienten hier allgemeine Verwaltungstätigkeiten als Vergleichsmaßstab. Im Vergleich zu diesen erhält die öffentliche Verwaltung in den Bereichen "Erziehung und Bildung", "IT und Telekommunikation", "Technische Verwaltung" und in sonstigen Tätigkeitsbereichen signifikant weniger Bewerbungen. Besonders stark ist dieser Effekt bei den IT-Berufen. Signifikant höhere Anzahlen an Bewerbungen gehen hingegen für Tätigkeiten im Bereich "Kunst und Kultur" sowie "Sicherheit und Ordnung" ein.

Auch zwei Marketinginstrumente haben einen Einfluss auf die Zahl der erhaltenen Bewerbungen. Auf Stellenanzeigen, die in privaten Stellenportalen (z. B. Stepstone oder Indeed) oder in Karriere-Portalen (z. B. Xing oder LinkedIn) veröffentlicht werden, gehen insgesamt mehr Bewerbungen ein. Diese Effekte sind jedoch nicht besonders stark ausgeprägt.

\subsection{Qualität des Pools an Bewerbungen}

Lediglich vier der untersuchten Faktoren haben einen Einfluss darauf, wie zufrieden die Verwaltungen mit dem Pool an Bewerbungen sind, den sie auf ihre Stellenanzeige erhalten haben. Bei Tätigkeiten im Bereich "IT und Telekommunikation" sowie "Technischer Verwaltung" sind die Befragten signifikant weniger zufrieden mit den erhaltenen Bewerbungen. Auch hier schlägt sich der starke Fachkräftemangel in diesen Bereichen nieder. Im Bereich der Presseund Öffentlichkeitsarbeit hingegen scheint die Bewerbungslage signifikant besser zu sein als bei allgemeinen Verwaltungstätigkeiten. Bemerkenswert ist außerdem, dass die Qualität der Bewerbungen höher zu sein scheint, wenn eine Anzeige in den sozialen Medien beworben wurde. Da dies nur ein Drittel der Befragten macht, ist hier ein Optimierungspotential zu erkennen. Einen umgekehrten Effekt haben Veröffentlichungen in Karriere-Portalen (z. B. Xing oder LinkedIn). Wird eine Anzeige dort veröffentlicht, sind die Teilnehmenden weniger zufrieden mit der Qualität der Bewerbungen. Möglicherweise wird dieses Instrument besonders dann genutzt, wenn bereits vorab abzusehen ist, dass es sehr schwierig wird, diese Position zu besetzen.

Unter den Faktoren, bei denen wir nicht besonders sicher sind, ob tatsächlich ein Effekt besteht, möchten wir außerdem die Anzahl von prosozialen Attributen in der Stellenanzeige hervorheben. Je mehr solcher Attribute in einer
In technischen und erzieherischen Berufen ist der Mangel an Bewerber:innen besonders stark

Bei technischen Berufen sind die Verantwortlichen besonders unzufrieden mit der Qualität der Bewerber:innen

Prosoziale Signale verbessern die Qualität des Pools an Bewerbungen 
Anzeige genannt wurden, desto eher waren die Befragten mit der Qualität der Bewerbungen zufrieden. Das Ergebnis muss zwar mit angemessener Vorsicht betrachtet werden, da Verbesserungen in diesem Bereich jedoch praktisch keine Kosten verursachen, könnten Stellenanzeigen hier problemlos optimiert werden.

\subsection{Qualität der ausgewählten Person}

Bei der Analyse der Zufriedenheit mit der ausgewählten Person finden sich fünf statistisch signifikante Einflussfaktoren. Offenbar macht hier auch die Ausgestaltung der Stellenanzeigen einen Unterschied. Sowohl die Anzahl der extrinsischen als auch der prosozialen Signale wirkt sich positiv auf die Zufriedenheit mit der ausgewählten Person aus. Stellt eine Anzeige viele Aspekte wie Flexibilität von Arbeitszeiten, Vereinbarkeit von Familie und Beruf, Urlaubstage oder Gehalt heraus, so führt dies offenbar zu einem Bewerber:innenfeld. aus dem sich eine noch geeignetere Person auswählen lässt. Dasselbe gilt für Attribute, die betonen, wie die Aufgabe dazu beiträgt, positiv auf das Leben anderer zu wirken und zum Gemeinwohl beitragen zu können. Festzustellen ist jedoch auch, dass der gefundene Effekt für prosoziale Motive stärker ausfällt.

Interessanterweise führen bürokratische Prozesse bei der Rekrutierung neuen Personals dazu, dass die Befragten letztlich weniger zufrieden mit der ausgewählten Person sind. Möglicherweise führen starre und als dysfunktional wahrgenommene Prozesse zu weniger optimalen Einstellungsentscheidungen. Die Bevölkerungsdichte am Einsatzort hat ebenfalls einen kleinen negativen Effekt auf die Zufriedenheit mit der ausgewählten Person. Je größer also die Bevölkerungsdichte, desto geringer die Zufriedenheit. Möglicherweise bekommen Verwaltungen in dichter besiedelten Gebieten, insbesondere in Großstädten, den Fachkräftemangel mit etwas Verspätung zu spüren und sind daher aktuell unzufriedener mit den Personen, die sie für eine Stelle gewinnen können. Überrascht hat uns zudem, dass die Befragten zufriedener mit der ausgewählten Person sind, wenn die entsprechende Stellenanzeige in einem Amtsblatt veröffentlicht wurde. Es ist nicht ganz klar, welcher Mechanismus diesen Effekt erklärt. Möglicherweise suchen verwaltungserfahrene Fachexperten in diesen speziellen Medien nach Stellenanzeigen oder es werden sehr spezifische Anzeigen in den Amtsblättern veröffentlicht.
Extrinsische und prosoziale Signale erhöhen die Zufriedenheit mit der ausgewählten Person

Übermäßige Bürokratie führt zu geringerem Rekrutierungserfolg 


\section{Handlungsempfehlungen}

Am Ende dieses Berichts möchten wir aus den präsentierten Ergebnissen einige Handlungsempfehlungen ableiten. Wir haben diese auch durch einige Aspekte ergänzt, die uns im Prozess der Datenerhebung und bei der Durchsicht vieler Stellenanzeigen aufgefallen sind.

\subsection{Wertschätzung}

- Kandidat:innen nicht das Gefühl geben, dass sie eine Last sind: In vielen Stellenanzeigen sowie insbesondere bei den automatischen Antworten, die auf eine Bewerbung folgen, vermitteln Verwaltungen den Eindruck, die Kandidat:innen halten die Verantwortlichen von wichtigeren Aufgaben ab. Hierzu gehört auch das weitverbreitete "Bitte kontaktieren Sie uns nicht; wir melden uns bei Ihnen". Fachkräfte, die zwischen einer Vielzahl von Arbeitgebern wählen können, sind durch derartige Botschaften schnell abgeschreckt. Versuchen Sie daher sowohl in der Stellenanzeige als auch in der Kommunikation Ihre Wertschätzung für die Bewerber:innen auszudrücken.

- Freundlichkeit: Einige Stellenanzeigen und automatische Antworten vermitteln einen eher unfreundlichen Eindruck. Diesen gilt es zu vermeiden.

\subsection{Erreichbarkeit}

- Mehrere Kommunikationswege eröffnen: $43 \%$ der von uns ursprünglich analysierten Stellenanzeigen enthielten - zumindest in den Metadaten - keine E-Mailadresse, an die Fragen zum Bewerbungsprozess hätten gesendet werden können. Umgekehrt war bei vielen Anzeigen keine Telefonnummer angegeben. Viele Menschen bevorzugen eine schriftliche oder mündliche Kontaktaufnahme und fühlen sich sehr unwohl, wenn sie auf die jeweils andere Form ausweichen müssen. Insbesondere die Kontaktaufnahme per E-Mail wird häufig als niederschwelliger empfunden und eröffnet damit die Möglichkeit, Fragen bezüglich einer Ausschreibung zu klären. Dies ist auch im Interesse der Verwaltung, die möglicherweise unpassende Bewerbungen vermeiden kann. Je nach Zielgruppe kann es sich auch auszahlen, alternative Kontaktmöglichkeit, beispielsweise über Social Media zu eröffnen.

- Kontaktperson klar benennen: Wenn eine Möglichkeit zur Kontaktaufnahme per E-Mail gegeben ist, ist es hilfreich, wenn auch eine konkrete Ansprechperson genannt und deren Geschlecht erkennbar ist. Ein "Dr. Maier" oder "ORR Müller" erschweren die Kontaktaufnahme unnötig.

- E-Mailadresse prüfen: In mehreren Fällen waren in den Stellenanzeigen E-Mailadressen als Kontaktmöglichkeit angegeben, die nicht existierten. Dies betraf insbesondere zentrale Adressen für Bewerbungen. Falls EMailadressen von individuellen Ansprechpartner:innen angegeben werden, sollten diese auch tatsächlich erreichbar sein.
Wertschätzende und freundliche Kommunikation

Einfach erreichbar sein 


\subsection{Professionalität}

- Schreibfehler vermeiden: Viele Rekrutierende erwarten, dass Bewerbungen fehlerfrei sind und unterstellen Bewerbungen mit Schreibfehlern mangelnde Ernsthaftigkeit oder Gewissenhaftigkeit. Diesen Maßstab sollten Verwaltungen auch an sich selbst anlegen. Es vermittelt keinen professionellen Eindruck, wenn die Stellenanzeige mehrere Schreibfehler enthält oder bereits der Titel der Anzeige oder der Behördenname falsch geschrieben sind.

\subsection{Sichtbarkeit}

- Stellenanzeigen auf vielen Wegen verbreiten: Auf Stellenportalen, die auf den öffentlichen Sektor spezialisiert sind, erreicht man oftmals hauptsächlich diejenigen Interessent:innen, die ohnehin bereits eine Tätigkeit in der Verwaltung anstreben. Auch die Behörden-Website hat in der Regel eine geringe Reichweite. Verwaltungen sollten überlegen, ob ein stärkeres Verbreiten der eigenen Stellenangebote möglich ist. Unsere Analysen zeigen, dass Anzeigen in privaten Stellenportalen (z.B. Stepstone oder Indeed) und auf Karriere-Portalen (z. B. Xing oder Linkedln) zu mehr Bewerbungen führen.

- Metadaten vollständig eintragen: In einigen Fällen waren die Metadaten, die bei bund.de zu Beginn der Stellenanzeige angezeigt werden, nicht vollständig ausgefüllt. Es fehlten beispielsweise Informationen über die Laufbahngruppe, die Art der Anstellung (Vollzeit oder Teilzeit) oder eine mögliche Befristung. Da potentielle Bewerber:innen diese Kriterien nutzen, um die Vielzahl der Anzeigen zu filtern, sollten Verwaltungen unbedingt alle verfügbaren Felder ausfüllen.

\subsection{Sprache}

- Verwaltungsjargon vermeiden: Manche Stellenanzeigen sind mit Begrifflichkeiten aus der Verwaltungssprache gespickt. Wenn Sie sich nicht absolut sicher sind, dass Sie ausschließlich Personen ansprechen wollen, die mit diesen Begrifflichkeiten bereits vertraut sind, sollten Sie Verwaltungsjargon vermeiden.

- Besoldungs- und Entgeltgruppen mit Beträgen hinterlegen: Viele potentielle Bewerber:innen sind mit dem Besoldungs- und Entgeltsystem des öffentlichen Sektors nicht vertraut. Dies trifft insbesondere auf jene zu, die nicht gezielt nach einer Anstellung im öffentlichen Dienst suchen. Diesen Personen hilft es, einen ungefähren Eindruck über die Höhe des Gehalts zu vermitteln. Diese Angabe kann gegebenenfalls nach Berufserfahrung aufgeschlüsselt oder als Minimal- und Maximalwerte angegeben werden.
Professionell auftreten

Anzeigen auf vielen Wegen verbreiten 


\subsection{Inhalte}

- Prosoziale Anreize herausstellen: Unsere Analysen zeigen, dass Verwaltungen davon profitieren, wenn sie in ihren Stellenanzeigen verdeutlichen, wie Stelleninhaber:innen durch ihre Tätigkeit einen Beitrag leisten und damit einen positiven Einfluss auf andere und das Gemeinwohl ausüben können. Diese Möglichkeit nutzen bisher nur wenige Arbeitgeber, obwohl dadurch die Zufriedenheit mit dem Pool an Bewerbungen sowie mit der ausgewählten Person gesteigert werden kann. Gerade die verwaltungswissenschaftliche Forschung der vergangenen Jahre unterstreicht, dass prosozial motivierte Mitarbeitende statistisch bessere Leistung erbringen und ein größeres Committment gegenüber ihrem Arbeitgeber zeigen.

- Extrinsische Anreize herausstellen: Stellenanzeigen, die viele extrinsische Anreize wie Urlaubstage, Betriebsrenten oder Weiterbildungsmöglichkeiten herausstellen, führen letztendlich zu einer größeren Zufriedenheit mit der ausgewählten Person. Es scheint daher lohnenswert, diese Vorzüge einer Stelle auch herauszustellen.

- Besondere extrinsische Anreize bieten: Viele extrinsische Anreize bieten kaum eine Möglichkeit, sich von anderen Arbeitgebern abzugrenzen. Wir haben jedoch immer wieder besondere Anreize in den Stellenanzeigen gefunden. Hierzu gehören beispielsweise das Vermitteln eines Kitaplatzes, die Möglichkeit zur kostenlosen Nutzung des öffentlichen Nahverkehrs, Supervision oder ein Fitness- und Sportprogramm.

\subsection{Prozess}

- Verfahren schnell abschließen: Stellenbesetzungsverfahren dauern oftmals sehr lange. Fachkräfte haben aber häufig Angebote von mehreren Arbeitgebern. Es ist daher wichtig, ein Verfahren schnell abschließen und ein Angebot unterbreiten zu können.

- Unnötige Bürokratie vermeiden: Regeln und vorgeschriebene Verfahrensweisen sind bei Stellenbesetzungen unvermeidbar. Nicht immer sind diese jedoch wirklich notwendig. Verwaltungen sollten prüfen, welche bürokratischen Hürden tatsächlich notwendig sind und wo sich Prozesse beschleunigen und flexibler gestalten lassen. Eine Digitalisierung des Rekrutierungsprozesses kann ebenfalls helfen und ermöglicht zudem schnellere Verfahren.
Prosoziale und extrinsische Motive betonen

Schnelle Verfahren erleichtern die Gewinnung von Fachkräften 


\section{Fazit}

Der öffentliche Sektor steht bei der Rekrutierung von neuem Personal vor großen Herausforderungen. Der Personalbedarf wird in den kommenden Jahren durch verstärkte Ruhestandseintritte noch weiter zunehmen. Gleichzeitig erfasst der Mangel an Fachkräften immer mehr Bereiche des Arbeitsmarkts und beschränkt sich nicht mehr alleine auf (informations-)technische Expert:innen.

Vor diesem Hintergrund kommt der Ausgestaltung und Kommunikation von Stellenanzeigen eine zentrale Rolle zu. Stellenanzeigen sind nach wie vor das zentrale Rekrutierungsinstrument der öffentlichen Verwaltung. Diese Studie zeigt, dass Verwaltungen dabei durchaus unterschiedliche Wege gehen. Dies beginnt bei der Verbreitung von Stellenanzeigen mithilfe verschiedener Marketinginstrumente und setzt sich bei den Inhalten der Anzeigen fort. Hierbei ist es insbesondere bemerkenswert, wie selten Verwaltungen betonen, dass ihre Beschäftigen einen wertvollen Beitrag für die Gesellschaft und das Gemeinwohl leisten. Diejenigen Verwaltung, die diese Chance nutzen, erfreuen sich an insgesamt qualifizierteren Bewerbungen und sind auch mit der ausgewählten Person zufriedener als andere.

Diese Studie wirbt damit für eine weitere Professionalisierung des Personalmanagements im öffentlichen Sektor und insbesondere der Personalgewinnung. Sie zeigt außerdem den Erkenntnisgewinn, den der Einsatz wissenschaftlicher Methoden bei der Personalgewinnung bieten kann und wie Verwaltungen und Wissenschaft durch Kooperationen neue Erkenntnisse gewinnen können.
Die Optimierung von Stellenausschreibungen kann den Rekrutierungserfolg steigern

\section{Forschungsteam}

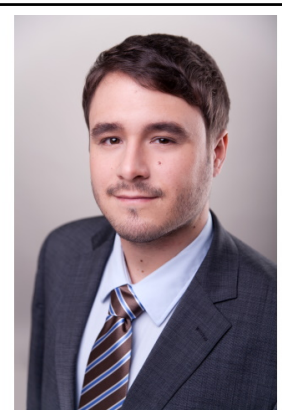

Prof Dr. Dominik Vogel Universität Hamburg Juniorprofessor für Public Management dominik.vogel@ wiso.uni-hamburg.de

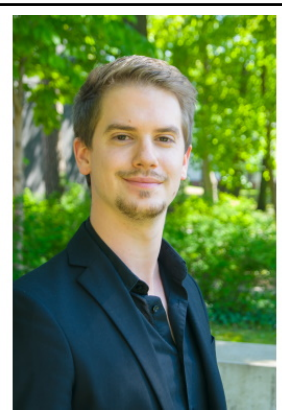

Prof. Dr. Matthias Döring Süddänische Universität Assistant Professor of Public Management mdoering@

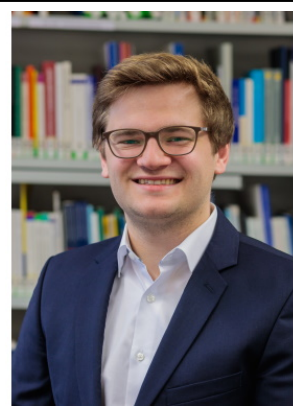

Martin Sievert, M.Sc.

Universität Mannheim

Wissenschaftlicher Mitarbeiter martin.sievert@ uni-mannheim.de 